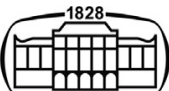

AKADÉMIAI KIADÓ

Journal of Psychedelic Studies

4 (2020) 3, 156-162

DOI:

$10.1556 / 2054.2020 .00126$

(C) 2020 The Author(s)

RESEARCH ARTICLE

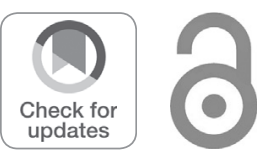

\title{
Behavioral effects of sub-anesthetic ketamine in a go/no-go task
}

\author{
ANDRÉ SEVENIUS NILSEN ${ }^{1}$, BJØRN ERIK JUEL ${ }^{1}$, \\ NADINE FARNES ${ }^{1}$, LUIS ROMUNDSTAD ${ }^{2}$ and \\ JOHAN FREDERIK STORM ${ }^{1, \dagger, *}$ (6)
}

\author{
${ }^{1}$ Department of Molecular Medicine, Institute of Basic Medical Sciences, University of Oslo, Oslo, \\ Norway \\ ${ }^{2}$ Department of Anesthesia, and Intervention Centre, Oslo University Hospital, Oslo, Norway
}

Received: December 13, 2019 • Accepted: August 23, 2020

Published online: September 24, 2020

\begin{abstract}
Background and aims: While psychedelic agents are known to have powerful, but largely unexplained, effects on contents of consciousness, there is an increasing interest in the potential clinical usefulness of such drugs for therapy, and legalization is discussed in some countries. Thus, it is relevant to study the effects of psychedelic compounds not only on experience, but also on behavioral performance. Methods: Seven healthy participants performed a motor response inhibition task before, during, and after subanesthetic doses of intravenously administered ketamine. The infusion rate was individually adjusted to produce noticeable subjective psychedelic effects. Results: We observed no statistically significant impact of sub-anesthetic ketamine on reaction times, omission errors, or post error slowing, relative to the preceding drug-free condition. However, we did observe significant correlations between performance impairment and self-reported, subjective altered states of consciousness, specifically experience of "anxiety" and "complex imagery." Conclusions: Considering the limited number of participants and large variation in strength of self-reported experiences, further studies with wider ranges of ketamine doses and behavioral tasks are needed to determine the presence and strength of potential behavioral effects.
\end{abstract}

\section{KEYWORDS}

psychedelics, ketamine, inhibition, go/no-go, behavior, phenomenology

\section{INTRODUCTION}

In recent years, there has been an increasing focus on psychedelic substances in both clinical trials and basic research, and several countries are relaxing or considering to relax the restrictions for both clinical and recreational use of psychedelics (Kyzar, Nichols, Gainetdinov, Nichols, \& Kalueff, 2017). In view of current and future increased availability of psychedelic substances, it is relevant to study how different psychedelic drugs affect various aspects of human behavior, cognitive abilities, and biology. Here we aim to test the effects of subanesthetic doses of ketamine on a classic behavioral inhibition task.

Ketamine affects the brain mainly through its action as a non-competitive $N$-methyl- $d$ aspartate (NMDA) antagonist (for an overview see Detsch \& Kochs, 1997). It is used as an anesthetic agent for certain procedures as it is strongly analgesic without blocking respiratory/circulatory systems (Sacchetti et al., 1994). At sub-anesthetic doses $(\leq 1.0 \mathrm{mg} / \mathrm{kg} / \mathrm{h}$; Schwenk et al., 2018), the drug can produce profound effects on subjective experience such as disembodiment, hallucinations, and synesthesia (e.g. Krystal et al., 1994; Morgan, Mofeez, Brandner, Bromley, \& Curran, 2004), and has for this reason been employed as a model of schizophrenia (Umbricht et al., 2000). In terms of behaviorally relevant physiological effects, sub-anesthetic ketamine has been found to cause enhanced recruitment of excitatory 
networks in motor cortex (Di Lazzaro et al., 2003), partially disrupt muscle contraction by affecting calcium binding at the neuromuscular junction (Marwaha, 1980), and increased pain inhibition at spinal and supraspinal sites by modifying pain signal propagation (Niesters et al., 2012). Sub-anesthetic doses of ketamine has also been observed to alter the brain's activity patterns and functional connectivity as assessed by functional magnetic resonance imaging imaging (fMRI; Scheidegger et al., 2012; Höflich et al., 2017), and functional and structural plasticity (Ly et al., 2018), as well as spectral properties as assessed by high-density electroencephalogram (EEG) (Vlisides et al., 2018). Among the observed cognitive effects of sub-anesthetic ketamine are impaired performance in goal maintenance and context processing (Umbricht et al., 2000), impaired memory functions, semantic processing, and procedural learning (Morgan et al., 2004), as well as reduced verbal fluency, vigilance, delayed recall, and other frontal lobe functions (Krystal et al., 1994; Radant, Bowdle, Cowley, Kharasch, \& Roy-Byrne, 1998). While these studies suggest widespread impaired behavioral performance, several studies observed no effect of subanesthetic ketamine on behavioral inhibition as measured by an anti-saccade task (Schmechtig et al., 2013; Steffens et al., 2018), and that attention and executive functioning remained largely spared (Morgan et al., 2004).

Given the above observations we might expect that subanesthetic ketamine should not impede behavioral performance on a standard go/no-go task, which measures behavioral inhibition (Littman \& Takács, 2017) and focused attention (Hong, Wang, Sun, Li, \& Tong, 2017), despite the mentioned heterogeneous changes in brain activity, cognition, behavior, and subjective experience. However, this apparent conflict in results might be explained by variance in individual subjective tolerance levels, ketamine doses, ketamine isomers, and infusion models between studies. Thus, we aimed to contribute to mapping the behavioral effects of sub-anesthetic doses of ketamine.

\section{METHODS}

Thirty-four participants were recruited through written adverts on the Oslo University Hospital notice boards. Ten participants completed the main study. For a detailed description of inclusion criteria, procedure, and overall study design, see Farnes, Juel, Nilsen, Romundstad, and Storm (2019). Briefly, racemic ketamine $(10 \mathrm{mg} / \mathrm{mL}$, Keta$\operatorname{lar}^{\circledR}$, Pfizer AS, Lysaker, Norway) was administered by an anesthesiologist or a nurse anesthetist. The drug was delivered by continuous intravenous infusion using an infusion pump (Braun ${ }^{\circledR}$ B. Braun Perfusor Space, B. Braun Melsungen AG, Melsungen, Germany). We started the infusion at a rate of $0.1 \mathrm{mg} / \mathrm{kg} / \mathrm{h}$ and increased by steps of $0.1 \mathrm{mg} / \mathrm{kg} / \mathrm{h}$. Participants were asked to relax and report spontaneously when they thought they had an effect of the drug (e.g. when they noticed perceptual disruptions or delusion-like ideas), and then again when they were sure they had an effect. Meanwhile, the anesthesiologist monitored physiological and behavioral signs (e.g. pulse, and speech patterns). Once a maximum continuous infusion of $1.0 \mathrm{mg} / \mathrm{kg} / \mathrm{h}$ was reached, or both the anesthesiologist and participant were certain that the participant had an effect of the drug the stepwise increase in dosage was stopped, and the infusion rate was stabilized at the maintenance dose for the remainder of the intervention. Thus, to account for individual differences in tolerance, the infusion levels were adjusted based on subjective reports primarily aiming to produce an altered state of consciousness consistent between participants.

Seven of the ten participants (participants 1 through 7 reported in the original study) also completed a behavioral inhibition test before (Pre), during (Int), and after (Post) ketamine administration. The remaining three participants did not perform the test due to a power analysis indicating that to reach significance given the observed effect sizes (after seven participants), we would need a sample size above that allowed by our ethical approval.

The behavioral inhibition test was a go/no-go task (Fig. 1) adapted from Verbruggen, Logan, and Stevens (2008). The task consisted of 150 trials (Fig. 2), where each trial started with a fixation point (200 ms $+0-333 \mathrm{~ms}$ jitter), then a target stimuli consisting of a white outline of either a diamond or a square shape on a gray background $(300 \mathrm{~ms})$. The trial ended at response or after $700 \mathrm{~ms}$. In $75 \%$ of the trials a square was presented (go trial) and participants were instructed to respond with a button press as fast as they could. In $25 \%$ of the trials a diamond was presented (no-go trial) and participants were instructed to then not respond. The order of presentation was randomized independently every session, thus the order of squares and diamonds was different each time the participant performed the task, and it was different for each participant. In order to reduce training effects, participants practiced the task on a previous occasion where inclusion and exclusion criteria were assessed in addition to establishing participant suitability to transcranial magnetic stimulation.

Sixty minutes after the end of ketamine administration, when all of the effects of ketamine had subsided, the participants rated their experience of the period during ketamine administration on an 11 dimensional altered states of consciousness questionnaire (11D-ASC; Dittrich, 1998).

Here we investigated three dependent variables; (1) mean reaction time (RT) in go trials, (2) fraction of omission errors (OMs) for no-go trials which is the response rate when one shouldn't respond, and, (3) post error slowing (PES) which is the mean RT of the go trial immediately following failure to inhibit response in a no-go trial. Note that due to the speed accuracy tradeoff between RT and OM, we adjusted OM with the slope of a linear regressor fitted between RT and OM. Measure (1) reflects overall processing speed, measure (2) reflects inhibitory control and sustained attention, and measure (3) reflects error detection and impulsiveness. As the procedure followed a A-B-A design (before, during, and after sub-anesthetic ketamine administration), intervention effects were analyzed with a repeated measures within-subjects analysis of variance (rmANOVA). 


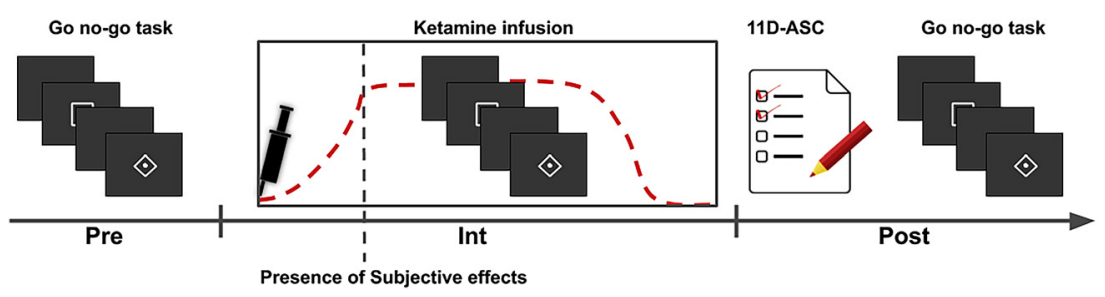

Fig. 1. Overview of experimental paradigm. First, participants performed the go/no-go task (Pre), followed by ketamine infusion increased in a stepwise manner until clear subjective effect (altered conscious perception), then the infusion rate was stabilized. During the sub-anesthetic ketamine state (Int), participants again performed the go/no-go task, after which the infusion was stopped. One hour later, participants answered the 11D-ASC questionnaire, and performed the go/no-go task again (Post)

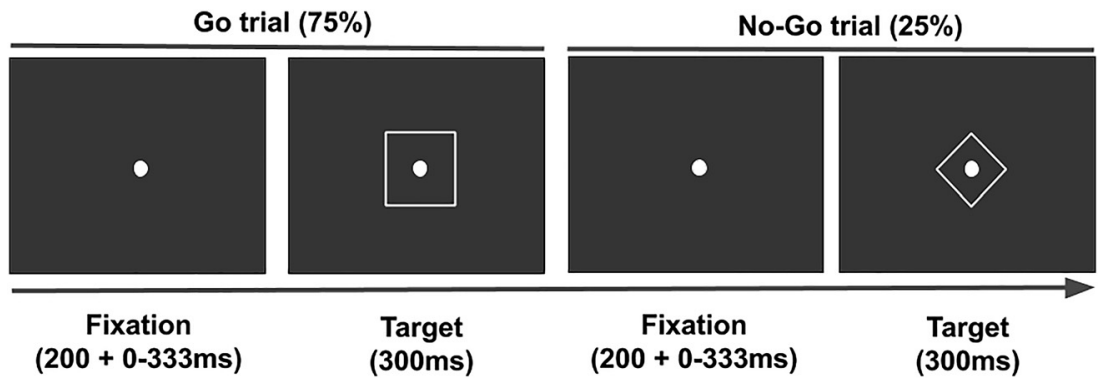

Fig. 2. Overview of the go/no-go task. First, a fixation cross was presented, followed by a target which was present up to $700 \mathrm{~ms}$ or until response. Participants were asked to respond as fast as possible when a square target was presented (go trial). Participants were instructed to not respond to diamond targets (no-go trial)

The subjective effects (measured with 11D-ASC) of ketamine administration, as well as total and stable maintenance dosage of ketamine given, were correlated with the degree of impairment (pre vs. intervention) of each dependent variable (RT, OM, PES) using Spearman's rank order correlation. Due to a low number of subjects relative to $11 \mathrm{D}$-ASC items and dependent variables, the tests were not adjusted for multiple comparisons.

The study was approved by the regional committees for medical and health research ethics (2015/1520/REK Sør-Øst A).

\section{RESULTS}

The average maintenance dose was $0.76 \mathrm{mg} / \mathrm{kg} / \mathrm{h}$ (SE = $0.065 \mathrm{mg} / \mathrm{kg} / \mathrm{h}$ ) and average total dosage given was $37.17 \mathrm{mg}$ $(\mathrm{SE}=5.56)$. For individual values and subjective reports on the 11D-ASC, see Table 1.

For the rmANOVA, we observed no significant sequence effect (i.e. linear) or an effect of intervention (i.e. quadratic), for either RT, OM, or PES (highest $F$ value reported): $F$ (2, 12) $<2.79, P>0.10, \eta^{2}<0.32$. While there was a tendency to

Table 1. 11D-ASC questionnaire results and ketamine dosages

\begin{tabular}{|c|c|c|c|c|c|c|c|c|c|}
\hline 11D-ASC & 1 & 2 & 3 & 4 & 5 & 6 & 7 & M & SE \\
\hline Experience of unity & 0.33 & 0.23 & 0.02 & 0.50 & 0.27 & 0.28 & 0.51 & 0.31 & 0.06 \\
\hline Spiritual experience & 0.35 & 0.01 & 0.01 & 0.33 & 0.10 & 0.00 & 0.15 & 0.14 & 0.06 \\
\hline Blissful state & 0.19 & 0.18 & 0.01 & 0.97 & 0.12 & 0.26 & 0.38 & 0.30 & 0.12 \\
\hline Insightfulness & 0.42 & 0.01 & 0.13 & 0.34 & 0.14 & 0.09 & 0.18 & 0.19 & 0.05 \\
\hline Disembodiment & 0.19 & 0.88 & 0.21 & 0.65 & 0.62 & 0.45 & 0.48 & 0.50 & 0.09 \\
\hline Impaired control and cognition & 0.12 & 0.21 & 0.10 & 0.36 & 0.39 & 0.06 & 0.20 & 0.21 & 0.05 \\
\hline Anxiety & 0.05 & 0.17 & 0.06 & 0.18 & 0.03 & 0.08 & 0.15 & 0.10 & 0.02 \\
\hline Complex imagery & 0.17 & 0.89 & 0.07 & 0.43 & 0.38 & 0.63 & 0.12 & 0.38 & 0.11 \\
\hline Elementary imagery & 0.01 & 0.99 & 0.01 & 0.78 & 0.78 & 0.91 & 0.16 & 0.52 & 0.17 \\
\hline Audio-visual synesthesia & 0.03 & 0.98 & 0.01 & 0.05 & 0.00 & 0.18 & 0.29 & 0.22 & 0.13 \\
\hline Changed meaning of percepts & 0.20 & 0.41 & 0.09 & 0.32 & 0.00 & 0.35 & 0.28 & 0.24 & 0.06 \\
\hline Global score & 0.18 & 0.40 & 0.07 & 0.42 & 0.25 & 0.26 & 0.26 & 0.26 & 0.05 \\
\hline \multicolumn{10}{|l|}{ Ketamine dosages } \\
\hline Total dose & 62.40 & 26.00 & 53.10 & 35.80 & 30.20 & 25.70 & 27.00 & 37.17 & 5.56 \\
\hline Maintenance dose & 1.00 & 0.60 & 1.00 & 0.70 & 0.70 & 0.70 & 0.60 & 0.76 & 0.065 \\
\hline
\end{tabular}

Self-reported 11D-ASC scores (relative to normal wakefulness) and recorded total dosage (mg/kg) and maintenance dosages (mg/kg/h) of ketamine for participants (1-7). 
Table 2. Descriptive statistics and rmANOVA results

\begin{tabular}{|c|c|c|c|c|c|c|c|c|c|}
\hline \multirow[b]{2}{*}{ Condition } & \multicolumn{3}{|c|}{ Reaction time } & \multicolumn{3}{|c|}{ Adj. omission errors } & \multicolumn{3}{|c|}{ Post error slowing } \\
\hline & Pre & Int & Post & Pre & Int & Post & Pre & Int & Post \\
\hline Mean & 311 & 314 & 300 & 1.01 & 1.07 & 0.94 & 15.17 & 32.45 & -4.64 \\
\hline$\pm \mathrm{SD}$ & 54.05 & 58.83 & 43.34 & 0.19 & 0.36 & 0.26 & 46.07 & 31.11 & 20.08 \\
\hline repeated measures ANOVA & $F$ & $p$ & $\eta^{2}$ & $F^{*}$ & $p$ & $\eta^{2}$ & $F$ & $p$ & $\eta^{2}$ \\
\hline & 1.39 & 0.29 & 0.19 & $0.47^{\star}$ & 0.54 & 0.07 & 2.79 & 0.10 & 0.32 \\
\hline
\end{tabular}

SD: Standard Deviation, $F$ : F-statistic of rmANOVA with degrees of freedom $(2,12), p: F$ associated $P$-value, $\eta^{2}$ : Partial Eta Squared.

${ }^{\star}$ Huynh-Feldt corrected, $F(1.16,6.94)$.

higher RT, OM, and PES during the intervention (Int), compared to before and after (Pre and Post), none of the effects were significant. See Table 2 and Fig. 3A, B, and C for an overview of results.

Introducing subjectively reported effects and dosages of ketamine, we observed significant correlations between maintenance dose of ketamine and degree of impaired behavior in RT and PES. In addition, there were significant correlations between the 11D-ASC sub dimensions anxiety and complex imagery, and OM and PES, respectively. See Fig. 3D.

Given that power in rmANOVA is dependent on the degree of correlation between repeated measures, we investigated test-retest reliability. We observed that for all dependent variables, pre and post was highly correlated (Spearman's $r_{\mathrm{s}}$ ), but intervention scores were not correlated with either pre or post for OM and PES. See Table 3. Given this apparent breakdown in test-retest reliability, we did a post-hoc rmANOVA with the factors anxiety and complex imagery from the 11D-ASC as covariates (non-corrected). The results showed that now there was a main effect of condition (pre, int, post) on $\mathrm{OM} F(2,8)=8.12, P=0.012$, $\eta^{2}=0.67$, and an interaction effect between condition and anxiety on $\mathrm{OM} ; F(2,8)=8.97, P=0.009, \eta^{2}=0.69$, as well as an interaction between condition and complex imagery on PES; $F(2,8)=4.91, P==0.041, \eta^{2}=0.55$. Further inspection showed that for OM, a subjective experience of more anxiety was associated with more errors, while a subjective experience of more complex imagery was associated with a higher degree of PES.

Finally, we investigated the required sample size given the observed $\eta^{2}$ for each main analysis, with $\mathrm{a}=0.05, \beta=$ 0.8 , and no sphericity correction analyzed with $G^{\star}$ Power 3.1.9.2 using the effect size specification by Cohen (2013).
A

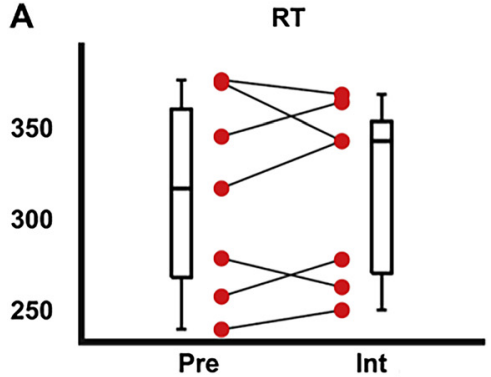

B

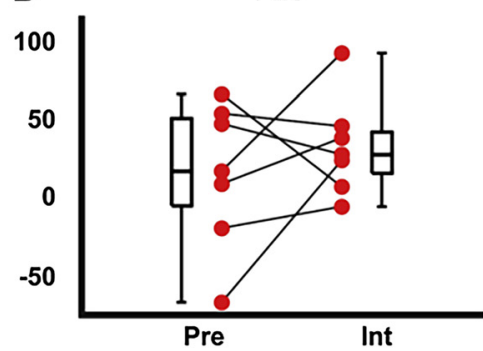

C OM

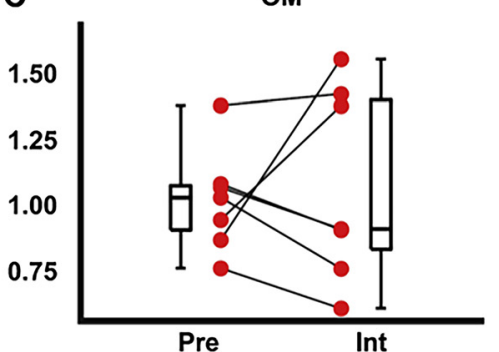

D

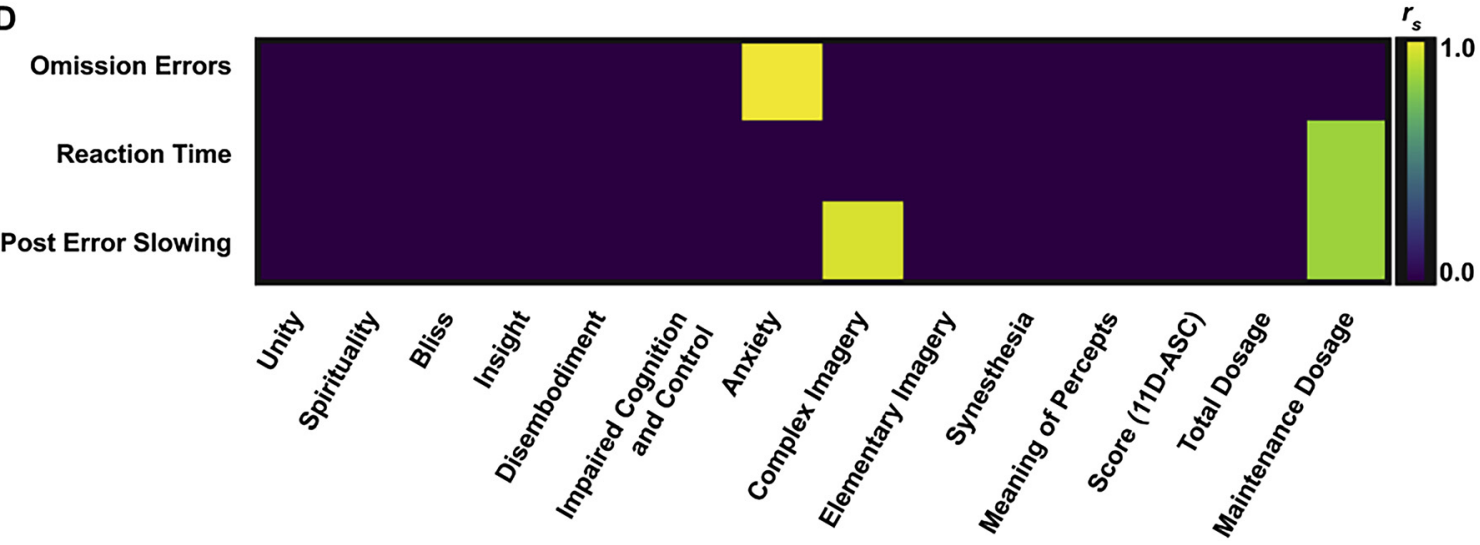

Fig. 3. Overview of results for pre-ketamine vs. intervention. (A) Reaction time (RT) in ms to go trials, (B) Post error slowing (PES), (C) Omission errors (failure to withhold response; OM) adjusted for speed-accuracy tradeoff, (D) Spearman rank order correlations between RT, OM, PES, sub-dimensions of 11D-ASC, and dosages of ketamine administered. Non-significant correlations are filtered out 
Table 3. Test-retest reliability

\begin{tabular}{lccc}
\hline$r_{\mathrm{s}}$ & Pre-int & Pre-post & Int-post \\
\hline RT & 0.93 & 0.93 & 0.79 \\
OM & 0.21 & 0.79 & 0.14 \\
PES & 0.21 & 0.86 & 0.32 \\
\hline
\end{tabular}

$r_{s}$ : Spearman rank-order correlation between conditions, RT: reaction time, OM: omission errors (adjusted), PES: post error slowing.

Required sample size for RT was $n=43$ (critical $F=3.1$ ), OM was $n=131$ (critical $F=130$ ), and PES was $n=23$ (critical $F=3.2$ ).

\section{DISCUSSION}

We observed no statistically significant effect of sub-anesthetic ketamine on performance on a go/no-go task. However, we did find that individual changes in omission errors (OMs) for no-go trials and post error slowing (PES) during ketamine administration were correlated with subjectively reported degrees of anxiety and complex imagery, respectively. In addition, both RT and PES were correlated with the maintenance level of ketamine administration. Post-hoc analysis showed that there was an effect of condition on OM and PES when controlling for the subscales anxiety and complex imagery in the 11-dimensional altered states of consciousness questionnaire (11D-ASC).

The main results did not show, at the group level, an overall impaired behavior due to sub-anesthetic ketamine administration, as seen in previous studies (e.g. Krystal et al., 1994; Morgan et al., 2004; Radant et al., 1998; Umbricht et al., 2000), although our results agreed with previous studies on response inhibition as measured by saccade inhibition (Schmechtig et al., 2013; Steffens et al., 2018), and attention as measured by the $n$-back task (Morgan et al., 2004).

There are several possible reasons for the main null findings. First, we fixed the infusion rate when it was determined that the subjects had an altered state of perception or behavior, as judged by both the anesthesiologist and the subjects themselves. This could have led to varying subjective psychotomimetic effects due to different subjective thresholds to report the presence of a definite effect even though it ensures at least the presence of an effect. This procedure also resulted in administration of heterogeneous total and maintenance dose (for more detail see Farnes et al., 2019). Thus, while we did not observe a significant impairment of behavior at the group level, we did see a strong correlation between $\mathrm{OM}$ and PES, and the anxiety and complex imagery subscales of the 11D-ASC, respectively, as well as between RT and PES, and the maintenance dose given. This could indicate that the lack of effect might be explained by variation in infusion rate, however, the average maintenance infusion rate $(M$ : 0.73 $[0.5-1.0] \mathrm{mg} / \mathrm{kg} / \mathrm{h}$ ) was on the higher end of what is characterized as sub-anesthetic ketamine (commonly 0.3-0.5 $\mathrm{mg} / \mathrm{kg} / \mathrm{h}$, up to the lower limit anesthetic rate of $1.0 \mathrm{mg} / \mathrm{kg} / \mathrm{h}$;
Schwenk et al., 2018). Secondly, while rmANOVA has decent power for seven subjects, it is on the lower end for the observed effect sizes. A post-hoc analysis given the observed results indicated that a required sample size on the lower end was $n=23$ for PES, and on the upper end $n=131$ for OM. Thus, while we could argue that performance monitoring as captured by PES could be affected by sub-anesthetic ketamine given an increased sample size, this is less likely for response inhibition as captured by OM. Thirdly, when controlling for the 11D-ASC subscales anxiety and complex imagery, which were strongly correlated with behavioral impairment, we observed significant effects of condition for both PES and OM. For OM in particular, the results indicated that those who experienced more relative anxiety during ketamine administration, had more errors, possibly due to stress, causing increased impulsivity. However, the relationship between PES and complex imagery was not so clear. In addition, considering that we did not adjust for multiple comparisons, the post-hoc analysis must be interpreted with caution. Fourth, the subjective effects might need to cross a certain threshold to be accompanied by behavioral impairment, especially for relatively easy tasks such as the go/no-go task. For example, for simpler tasks, participants under sub-anesthetic ketamine might be able to compensate via increased focus or other recruitment of neural resources (Steffens et al., 2018). Finally, the study was not placebo-controlled. While it seems unlikely that use of placebo would have substantially altered the results, since participants are generally very accurate in guessing conditions in placebo-controlled studies of effects of psychedelics (e.g. Morgan et al., 2004), a placebo control might have revealed a training effect due to repeated performance. The results do suggest a small behavioral increase from Pre- to Post-intervention, and a single prior practice run as performed in this study is generally not assumed to fully saturate task learning (e.g. Benikos, Johnstone, \& Roodenrys, 2013). However, the breakdown in intercondition performance correlation on $\mathrm{OM}$ in particular, suggests that performance during sub-anesthetic ketamine is largely independent of performance before and after intervention.

In conclusion, while we did observe indications of an effect of sub-anesthetic doses of ketamine on go/no-go task performance, no statistically significant overall effect was observed at the group level. However, the results must be interpreted in light of several limitations of this study. Thus, there remains a need for future studies to test how subanesthetic doses of ketamine (and other substances with psychotomimetic effects) affect behavior and cognition, and how long-lasting these effects are. The study of behavioral effects during psychedelics may be improved further by using placebo and several rounds of pre-intervention practice in order to ensure performance saturation before testing.

Ethical statement: The study was approved by the regional committees for medical and health research ethics (2015/ 1520/REK Sør-Øst A). All authors report no conflict of interest. All participants gave their written consent to participate in the study. 
Author contributions: ASN: conceived of study, data acquisition, performed experiment, analysis, interpretation, and wrote the manuscript. BEJ: data acquisition, performed experiment, interpretation, revised manuscript. NF: data acquisition, performed experiment, revised manuscript. LR: data acquisition, performed experiment, revised manuscript JFS: conceived of study, interpretation, revised manuscript.

\section{ACKNOWLEDGEMENTS}

We thank Franz Vollenveider for advice regarding ketamine administration, Brita Noorland for help with ketamine administration, and Anikó Kuztor for helping with the data acquisition. This study was supported by the European Union's Horizon 2020 research and innovation programme under grant agreement 7202070 (Human Brain Project; HBP) and the Norwegian Research Council (NRC: 262950/ F20 and 214079/F20). The authors report no conflict of interest.

\section{REFERENCES}

Benikos, N., Johnstone, S. J., \& Roodenrys, S. J. (2013). Short-term training in the Go/Nogo task: Behavioural and neural changes depend on task demands. International Journal of Psychophysiology: Official Journal of the International Organization of Psychophysiology, 87(3), 301-312. https://doi.org/10.1016/j. ijpsycho.2012.12.001.

Cohen, J. (2013). Statistical power analysis for the behavioral sciences. Academic Press.

Detsch, O., \& Kochs, E. (1997). Effects of ketamine on CNSfunction. Der Anaesthesist, 46(Suppl. 1), S20-S29. Retrieved from https://www.ncbi.nlm.nih.gov/pubmed/9163274.

Di Lazzaro, V., Oliviero, A., Profice, P., Pennisi, M. A., Pilato, F., Zito, G., et al. (2003). Ketamine increases human motor cortex excitability to transcranial magnetic stimulation. The Journal of Physiology, 547(Pt 2), 485-496. https://doi.org/10.1113/jphysiol. 2002.030486.

Dittrich, A. (1998). The standardized psychometric assessment of altered states of consciousness (ASCs) in humans. Pharmacopsychiatry, 31(Suppl. 2), 80-84. https://doi.org/10.1055/s2007-979351.

Farnes, N., Juel, B. E., Nilsen, A. S., Romundstad, L., \& Storm, J. F. (2019). Increased signal diversity/complexity of spontaneous EEG in humans given sub-anaesthetic doses of ketamine. bioRxiv. Retrieved from https://www.biorxiv.org/content/early/ 2019/01/02/508697.abstract.

Höflich, A., Hahn, A., Küblböck, M., Kranz, G. S., Vanicek, T., Ganger, S., et al. (2017). Ketamine-dependent neuronal activation in healthy volunteers. Brain Structure \& Function, 222(3), 1533-1542. https://doi.org/10.1007/s00429-0161291-0.

Hong, X., Wang, Y., Sun, J., Li, C., \& Tong, S. (2017). Segregating top-down selective attention from response inhibition in a spatial cueing Go/NoGo task: An ERP and source localization study. Scientific Reports, 7(1), 9662. https://doi.org/10.1038/ s41598-017-08807-z.

Krystal, J. H., Karper, L. P., Seibyl, J. P., Freeman, G. K., Delaney, R., Bremner, J. D., et al. (1994). Subanesthetic effects of the noncompetitive NMDA antagonist, ketamine, in humans. Psychotomimetic, perceptual, cognitive, and neuroendocrine responses. Archives of General Psychiatry, 51(3), 199-214. https://doi.org/10.1001/archpsyc.1994.03950030035004.

Kyzar, E. J., Nichols, C. D., Gainetdinov, R. R., Nichols, D. E., \& Kalueff, A. V. (2017). Psychedelic drugs in biomedicine. Trends in Pharmacological Sciences, 38(11), 992-1005. https://doi.org/ 10.1016/j.tips.2017.08.003.

Littman, R., \& Takács, Á. (2017). Do all inhibitions act alike? A study of go/no-go and stop-signal paradigms. PloS One, 12(10), e0186774. https://doi.org/10.1371/journal.pone.0186774.

Ly, C., Greb, A. C., Cameron, L. P., Wong, J. M., Barragan, E. V., Wilson, P. C., et al. (2018). Psychedelics promote structural and functional neural plasticity. Cell Reports, 23(11), 3170-3182. https://doi.org/10.1016/j.celrep.2018.05.022.

Marwaha, J. (1980). Some mechanisms underlying actions of ketamine on electromechanical coupling in skeletal muscle. Journal of Neuroscience Research, 5(1), 43-50. https://doi.org/ 10.1002/jnr.490050107.

Morgan, C. J. A., Mofeez, A., Brandner, B., Bromley, L., \& Curran, H. V. (2004). Acute effects of ketamine on memory systems and psychotic symptoms in healthy volunteers. Neuropsychopharmacology: Official Publication of the American College of Neuropsychopharmacology, 29(1), 208-218. https://doi. org/10.1038/sj.npp.1300342.

Niesters, M., Khalili-Mahani, N., Martini, C., Aarts, L., van Gerven, J., van Buchem, M. A., et al. (2012). Effect of subanesthetic ketamine on intrinsic functional brain ConnectivityA placebocontrolled functional magnetic resonance imaging study in healthy male volunteers. Anesthesiology, 117(4), 868-877. https://doi.org/10.1097/ALN.0b013e31826a0db3.

Radant, A. D., Bowdle, T. A., Cowley, D. S., Kharasch, E. D., \& RoyByrne, P. P. (1998). Does ketamine-mediated N-methyl-Daspartate receptor antagonism cause schizophrenia-like oculomotor abnormalities? Neuropsychopharmacology: Official Publication of the American College of Neuropsychopharmacology, 19(5), 434444. https://doi.org/10.1016/S0893-133X(98)00030-X.

Sacchetti, A., Schafermeyer, R., Geradi, M., Graneto, J., Fuerst, R. S., Cantor, R., et al. (1994). Pediatric analgesia and sedation. Annals of Emergency Medicine, 23(2), 237-250. https://doi.org/ 10.1016/s0196-0644(94)70037-0.

Scheidegger, M., Walter, M., Lehmann, M., Metzger, C., Grimm, S., Boeker, H., et al. (2012). Ketamine decreases resting state functional network connectivity in healthy subjects: Implications for antidepressant drug action. PloS One, 7(9), e44799. https://doi.org/10.1371/journal.pone.0044799.

Schmechtig, A., Lees, J., Perkins, A., Altavilla, A., Craig, K. J., Dawson, G. R., et al. (2013). The effects of ketamine and risperidone on eye movement control in healthy volunteers. Translational Psychiatry, 3, e334. https://doi.org/10.1038/tp.2013.109.

Schwenk, E. S., Viscusi, E. R., Buvanendran, A., Hurley, R. W., Wasan, A. D., Narouze, S., et al. (2018). Consensus guidelines on the use of intravenous ketamine infusions for acute pain management from the American Society of Regional Anesthesia 
and Pain Medicine, the American Academy of Pain Medicine, and the American Society of Anesthesiologists. Regional Anesthesia \& Pain Medicine, 43(5), 456-466.

Steffens, M., Neumann, C., Kasparbauer, A.-M., Becker, B., Weber, B., Mehta, M. A., et al. (2018). Effects of ketamine on brain function during response inhibition. Psychopharmacology, 235(12), 35593571. https://doi.org/10.1007/s00213-018-5081-7.

Umbricht, D., Schmid, L., Koller, R., Vollenweider, F. X., Hell, D., \& Javitt, D. C. (2000). Ketamine-induced deficits in auditory and visual context-dependent processing in healthy volunteers: Implications for models of cognitive deficits in schizophrenia.
Archives of General Psychiatry, 57(12), 1139-1147. https://doi. org/10.1001/archpsyc.57.12.1139.

Verbruggen, F., Logan, G. D., \& Stevens, M. A. (2008). STOP-IT: Windows executable software for the stop-signal paradigm. Behavior Research Methods, 40(2), 479-483. https://doi.org/10. 3758/BRM.40.2.479.

Vlisides, P. E., Bel-Bahar, T., Nelson, A., Chilton, K., Smith, E., Janke, E., et al. (2018). Subanaesthetic ketamine and altered states of consciousness in humans. British Journal of Anaesthesia, 121(1), 249-259. https://doi.org/10.1016/j.bja.2018.03. 011. 